\title{
Influence of Annealing on the Electrical Properties of Cz-Si Wafers Previously Subjected to the Hydrogen Ion-Beam Treatment
}

\author{
A. Fedotov ${ }^{a, *}$, O. Korolik ${ }^{a}$, A. MazaniK $^{a}$, T. Koltunowicz ${ }^{b}$ And P. Żukowski ${ }^{b}$ \\ ${ }^{a}$ Belarussian State University, 4, Nezavisimosti St., 220030 Minsk, Belarus \\ ${ }^{b}$ Lublin University of Technology, Nadbystrzycka 38, 20-618 Lublin, Poland
}

The main goal of this work is to establish the influence of annealing on the properties of Cz-Si wafers previously subjected to the hydrogen ion-beam treatment at 25 or $300-350{ }^{\circ} \mathrm{C}$. It is demonstrated by the conducted study that, despite similarity in the effects of the hydrogen ion-beam treatment at different temperatures on some electrical properties of the wafers (photovoltage spectra, thermoelectromotive force sign), thermal stability of changes in these properties due to the hydrogen ion-beam treatment depends on the hydrogenation temperature.

PACS: 61.72.uf, 81.40.Ef

\section{Introduction}

It is well known that the introduction of hydrogen into silicon lattice influences drastically the defect ensemble of material [1]. Some physical effects proceeding in hydrogenated silicon have found practical application (defect passivation [2], silicon layer splitting in Smart-Cut process [3], etc.). It is also known that the radiation hardness of silicon can be significantly modified by hydrogenation.

One of the most commonly used hydrogenation methods is the hydrogen ion-beam treatment. In spite of the fact that during such treatment ions are of a relatively low energy (as a rule, no more than a few $\mathrm{keV}$ ), the effect of the ion beam is liable to change the material electrical properties. Therefore, it is necessary to take into account the defect formation caused by the low-energy ion beam for better understanding of the influence exerted by the plasma introduced hydrogen on the properties of silicon. This can be partly done by a study of the influence of annealing on the electrophysical parameters of silicon wafers previously subjected to the hydrogen ion-beam treatment.

\section{Experimental}

The standard commercial $0.5-40 \Omega \mathrm{cm}$ both $n$ - and $p$-type $\mathrm{Cz}$-Si wafers were subjected to the hydrogen ion-beam treatment at 25 or $300-350^{\circ} \mathrm{C}$. The ion energy was equal to $300 \mathrm{eV}$, current density $-0.15 \mathrm{~mA} / \mathrm{cm}^{2}$, duration of treatment $-30 \mathrm{~min}$. The ion-treated samples were annealed in an inert ambient (argon) gas during 30 min at a temperature ranging from 200 to $900^{\circ} \mathrm{C}$.

The electrical properties of the samples studied were investigated using the spectral dependences of photovoltage: thermoelectromotive force (thermoEMF) and

* corresponding author; e-mail: fedotov@bsu.by

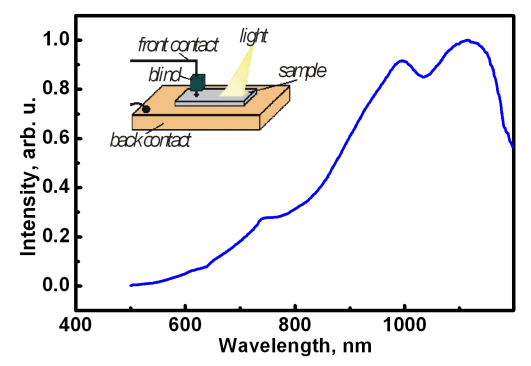

Fig. 1. Spectrum of the used lamp and the photovoltage measurement scheme (in the inset).

capacitance-voltage methods. Photovoltage spectra were recorded using a grid monochromator in the spectral range $500-1200 \mathrm{~nm}$ with a spectral resolution of $5 \mathrm{~nm}$. Let us note that all the presented photovoltage spectra are not normalized to the spectrum for the used lamp given in Fig. 1. The scheme of photovoltage measurements is presented in the inset of Fig. 1. The transversal (when the applied electric field is normal to the wafer plane) capacitance-voltage $(C-V)$ characteristics were measured using the Agilent-E4980A LCR-meter. In these experiments the ohmic contact was formed on the back (untreated) surface of the sample, and the clamping contact was applied to the front (ion treated) one (inset in Fig. 3a). The amplitude and frequency of the testing signal were equal to $40 \mathrm{mV}$ and $100 \mathrm{kHz}$, respectively.

\section{Results and discussion}

In Table the results of the thermoEMF sign measurements for the hydrogen ion-beam treated and subsequently annealed $12 \Omega \mathrm{cm}$-type $\mathrm{Cz}$-Si wafers are listed. As can be seen from Table, due to the hydrogen ion-beam treatment at both 25 and $300-350^{\circ} \mathrm{C}$, a sign of thermoEMF at the treated surface is changed from "+" 
to "-". However, the influence of annealing on the thermoEMF sign depends on the ion-beam treatment temperature: in the case of ion-beam hydrogenation at room temperature a positive sign of thermoEMF is restored as a result of annealing already at $200^{\circ} \mathrm{C}$, whereas for the wafers hydrogenated at $300-350^{\circ} \mathrm{C}$ a negative sign of thermoEMF is retained after annealing at the temperatures up to $450^{\circ} \mathrm{C}$. The same results are obtained for the $p$-type wafers with higher resistivity $(40 \Omega \mathrm{cm})$. Let us note that for the $n$-type wafers a negative thermoEMF sign is retained after both ion-beam hydrogenation and annealing.

TABLE

ThermoEMF sign measured at the front and back surfaces of the $12 \Omega \mathrm{cm}$-type $\mathrm{Cz}$-Si wafer after the hydrogen ion-beam treatment (designated as $\mathrm{H}$ ) and subsequent annealing at $200^{\circ} \mathrm{C}(\mathrm{A}-200), 350^{\circ} \mathrm{C}(\mathrm{A}-350), 450{ }^{\circ} \mathrm{C}$ (A-450), and $600^{\circ} \mathrm{C}(\mathrm{A}-600)$.

\begin{tabular}{c|c|c|c|c}
\hline \hline \multirow{2}{*}{ Sample } & \multicolumn{3}{|c|}{ Ion-beam treatment temperature [ $\left.{ }^{\circ} \mathrm{C}\right]$} \\
\cline { 2 - 5 } & \multicolumn{2}{|c|}{25} & \multicolumn{2}{c}{$300-350$} \\
\cline { 2 - 5 } & Sample surface & \multicolumn{2}{c}{ Sample surface } \\
\cline { 2 - 5 } & Front & Back & Front & Back \\
\hline H & - & + & - & + \\
A-200 & + & + & - & + \\
A-350 & + & + & - & + \\
A-450 & + & + & - & + \\
A-600 & + & + & + & +
\end{tabular}

Figure 2 presents the photovoltage spectra for the $12 \Omega \mathrm{cm} p$-type $\mathrm{Cz}$-Si wafers subjected to hydrogen ion-beam treatment at $315^{\circ} \mathrm{C}$ and subsequent annealing with illumination of the front (Fig. 2a) and back (Fig. 2b) surfaces. The sign and spectral shape of photovoltage for the ion-beam treated wafers point to the appearance of the potential barrier on the treated surface. Annealing at $200-450{ }^{\circ} \mathrm{C}$ leads to an insignificant increase of the signal magnitude and also to the shift of maxima to the longand short wavelength regions at illumination of the front and back surfaces, respectively. As the light penetration depth increases with the wavelength, such transformation of photovoltage spectra exhibits an increase in the depth of the $p-n$-junction formed due to the ion-beam treatment. As can be seen, the annealing at $600^{\circ} \mathrm{C}$ leads to disappearance of the barrier photovoltage, that correlates well with the thermoEMF measurements. The similar results were observed for the $40 \Omega \mathrm{cm}$-type $\mathrm{Cz}-\mathrm{Si}$ wafers.

The experiments have shown that the barrier photovoltage arising in the $p$-type $\mathrm{Cz}$-Si due to the hydrogen ion-beam treatment at $25^{\circ} \mathrm{C}$ disappears after the annealing performed at a minimal temperature $\left(200^{\circ} \mathrm{C}\right)$ in agreement with the changing thermoEMF sign for these samples.

No barrier photovoltage was fixed for the $n$-type wafers both after the ion-beam treatment and subsequent annealing (these spectra are not shown here).



Fig. 2. Photovoltage spectra for the $12 \Omega \mathrm{cm} p$-type $\mathrm{Cz}-\mathrm{Si}$ subjected to the hydrogen ion-beam treatment at $315^{\circ} \mathrm{C}$ (curve 1 ) and subsequent annealing at $200{ }^{\circ} \mathrm{C}$ (curve 2), $350{ }^{\circ} \mathrm{C}$ (curve 3), $450^{\circ} \mathrm{C}$ (curve 4), and $600^{\circ} \mathrm{C}$ (curve 5) with illumination of the front (a) and back (b) surfaces.

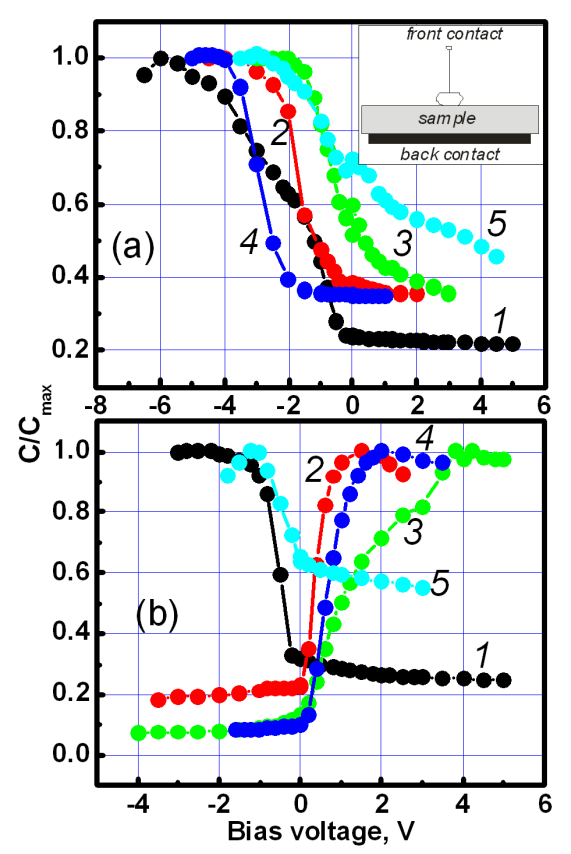

Fig. 3. Transversal capacitance-voltage characteristics for the $12 \Omega \mathrm{cm} p$-type $\mathrm{Cz}$-Si wafers subjected to the hydrogen ion-beam treatment (curve 1) and subsequent annealing at $200{ }^{\circ} \mathrm{C}$ (curve 2), $350{ }^{\circ} \mathrm{C}$ (curve 3), $450{ }^{\circ} \mathrm{C}$ (curve 4), and $600{ }^{\circ} \mathrm{C}$ (curve 5); (a) hydrogenation at $25^{\circ} \mathrm{C}$; (b) hydrogenation at $315^{\circ} \mathrm{C}$; the measurement scheme is given in the inset. 
Figure 3 shows the measured transversal capacitancevoltage characteristics for the $12 \Omega \mathrm{cm}$ p-type $\mathrm{Cz}-\mathrm{Si}$ wafers. As can be seen, the shape of $C-V$ curves is qualitatively similar to the shape observed for the MOS structures, that is explained by the formation of a thin (several nanometers) oxide layer on the ion-treated surface [4]. In so doing $C-V$ curves for the $p$-type wafers subjected to the hydrogen ion-beam treatment at $25^{\circ} \mathrm{C}$ correspond to $C-V$ for the MOS structure formed on the $p$-type substrate. At the same time, the $C-V$ shape for the wafers hydrogenated at $300-350^{\circ} \mathrm{C}$ after annealing at $200-450{ }^{\circ} \mathrm{C}$ is similar to that for the MOS structure on the $n$-type substrate, and the initial shape of $C-V$ is restored only after annealing at $600^{\circ} \mathrm{C}$. Let us note that in the case of the $40 \Omega \mathrm{cm} p$-type $\mathrm{Cz}$-Si wafers the $C-V$ curves for the MOS structure on the $n$-type substrate are observed already after the ion-beam treatment at $300-350^{\circ} \mathrm{C}$, being retained after annealing at $200-450{ }^{\circ} \mathrm{C}$. It is seen from Fig. 3 that annealing leads to a change in the ratio of maximal and minimal capacitances as well as to the shift of $C-V$ curves along the voltage axis. This can be explained by changes of the impurity concentration in the near-surface layer as well as by the density and energy distribution of the surface electronic states. However, as the structure of the near-surface layer in the samples under study is more complex compared to "dielectric-semiconductor", it is difficult to perform a quantitative analysis of the measured $C-V$ curves.

Let us analyze the obtained results. Based on the performed experiments, we can conclude that, despite similar influence of the hydrogen ion-beam treatment at $25-350^{\circ} \mathrm{C}$ on some electrical properties (thermoEMF sign, photovoltage spectra) of the $p$-type $\mathrm{Cz}$-Si wafers, mechanisms of the influence on these properties in the case of hydrogenation at room and at elevated $\left(300-350^{\circ} \mathrm{C}\right)$ temperatures are different. The $C-V$ characteristics for the MOS structure on the $n$-type substrate observed for the $p$-type wafers are associated with the formation of shallow donors in the near-surface region. A thickness of this overcompensated region comes to, at least, several hundreds of nanometers (typical space-charge region thickness) that is much greater compared to the projective range $R_{p}$ of hydrogen ions (a few nanometers). It is known that the introduction of hydrogen into silicon can lead to the formation of shallow hydrogen donors (SHD) [5]. However, in our case these donors cannot be responsible for the observed transformation in the electrical properties of $\mathrm{Cz}$-Si wafers because for their formation the radiation defects are required and hence they can arise only within the projective range of hydrogen ions. It can be stated that the observed silicon overcompensation to the $n$-type is determined by the hydrogen enhanced formation of shallow thermal donors (STD) [6]. The observed increase of the $p-n$ junction depth under annealing (Fig. 2) is naturally explained by the additional formation of STDs in the hydrogenated material.

The changes in the electrical properties of the wafers subjected to the hydrogen ion-beam treatment performed at room temperature (change of thermoEMF sign, appearance of barrier photovoltage, restoring of $C-V$ shape) were explained in [7] by the following cooperative processes: (i) ion-induced formation of a positive surface charge; (ii) boron deactivation by the generated interstitial silicon atoms; (iii) silicon amorphization and hence formation of the barrier "a-Si/c-Si" heterostructure. As follows from the presented results, these changes can be eliminated by annealing at $200^{\circ} \mathrm{C}$.

\section{Conclusion}

The performed study makes it possible to draw the following conclusions:

1. Hydrogen ion-beam treatment both at room and at elevated $\left(300-350^{\circ} \mathrm{C}\right)$ temperatures has a similar influence on electrical properties of the $p$-type $\mathrm{Cz}$-Si wafers, leading to the changed sign of thermoEMF and to the appearance of the barrier photovoltage on the treated surface.

2. Thermal stability of the properties of the hydrogen ion-beam treated $p$-type wafers depends on the treatment temperature: the initial (pretreatment) properties of the wafers hydrogenated at $25^{\circ} \mathrm{C}$ are restored as a result of annealing at $200^{\circ} \mathrm{C}$, whereas for the wafers hydrogenated at $300-350^{\circ} \mathrm{C}$ annealing at $200-450{ }^{\circ} \mathrm{C}$ leads to the increased depth of the formed $p-n$ junction, the initial properties being restored only after annealing at $600{ }^{\circ} \mathrm{C}$.

3. The obtained results point to the differences in the processes under study when the hydrogen ion-beam treatment is realized at room and at elevated temperatures: with the room temperature treatment the changes in the electrical properties of wafers are determined by the ion-beam stimulated defect formation, whereas the formation of shallow donors determines the influence of the ion-beam treatment performed at elevated temperatures.

\section{References}

[1] S.J. Pearton, J.W. Corbett, M. Stavola, Hydrogen in Crystalline Semiconductors, Springer Verlag, Berlin 1992.

[2] R. Luedemann, Mater. Sci. Eng. B 58, 86 (1999).

[3] M. Bruel, Nucl. Instrum. Methods Phys. Res. B 108, 313 (1996).

[4] O. Zinchuk, A. Saad, N. Drozdov, A. Fedotov, S. Kobeleva, A. Mazanik, A. Patryn, V. Pilipenko, A. Pushkarchuk, J. Mater. Sci. Mater. Electron. 19, 273 (2008).

[5] Y. Ohmura, Y. Zohta, M. Kanazawa, Phys. Status Solidi A 15, 93 (1973).

[6] H.J. Stein, S. Hahn, Appl. Phys. Lett. 56, 63 (1990).

[7] O. Zinchuk, N. Drozdov, A. Fedotov, A. Mazanik, N. Krekotsen, V. Ukhov, Vacuum 83, S99 (2009). 